Article

\title{
Stochastic Drone Fleet Deployment and Planning Problem Considering Multiple-Type Delivery Service
}

\author{
Ming Liu ${ }^{1}{ }^{1}$, Xin Liu $^{1}$, Maoran Zhu ${ }^{1, *}$ and Feifeng Zheng ${ }^{2}$ \\ 1 School of Economics \& Management, Tongji University, Shanghai 200092, China \\ 2 Glorious Sun School of Business \& Management, Donghua University, Shanghai 200051, China \\ * Correspondence: 1531140@tongji.edu.cn
}

Received: 21 May 2019; Accepted: 9 July 2019; Published: 16 July 2019

\begin{abstract}
Drone delivery has a great potential to change the traditional parcel delivery service in consideration of cost reduction, resource conservation, and environmental protection. This paper introduces a novel drone fleet deployment and planning problem with uncertain delivery demand, where the delivery routes are fixed and couriers work in collaboration with drones to deliver surplus parcels with a relatively higher labor cost. The problem involves the following two-stage decision process: (i) The first stage determines the drone fleet deployment (i.e., the numbers and types of drones) and the drone delivery service module (i.e., the time segment between two consecutive departures) on a tactical level, and (ii) the second stage decides the numbers of parcels delivered by drones and couriers on an operational level. The purpose is to minimize the total cost, including (i) drone deployment and operating cost and (ii) expected labor cost. For the problem, a two-stage stochastic programming formulation is proposed. A classic sample average approximation method is first applied. To achieve computational efficiency, a hybrid genetic algorithm is further developed. The computational results show the efficiency of the proposed approaches.
\end{abstract}

Keywords: drone; fleet deployment; service module; parcel delivery

\section{Introduction}

With the development of sensing, computing, cognitive radio, and robotic technologies, the application of drones, originated from military industry, is rapidly extending to service, agriculture, public safety networks (Sikeridis et al. [1]), and healthcare. It is recognized that drones have a great potential to change the traditional express transportation, due to the fact that (i) a drone can respond rapidly to demand and its delivery is not restricted by the road conditions (Hong et al. [2]); (ii) drone delivery will be more cost-effective to get to places where traditional transportation modes would be difficult to reach (Zhang and Kovacs [3]); (iii) employing drones in a delivery system is more environmentally friendly, as drones are powered by electricity and thus lead to less greenhouse gas emissions. JingDong (i.e., JD.com), China's second largest e-commerce giant, claimed that deliveries in underdeveloped rural areas can cost up to six times more than city trips and, thus, the usage of drones can reduce the delivery cost by 70 percent or more (Aleem [4]).

Drone delivery grows very fast in the service and transportation industry. Amazon founder Jeff Bezos first announced that Amazon has developed a fleet of drones to deliver lightweight commercial products in 2013 (Rose [5]). JD.com in China had seven types of delivery drones in testing or operation across four provinces in 2017 (Aleem [4]). Their drones are now able to delivery packages weighing between 5 to $30 \mathrm{~kg}$, and the maximum travel distance is about $200 \mathrm{~km}$ before recharging. Moreover, Ele.me, one of the largest food delivery companies in China, announced that they have developed a fleet of drones to deliver food in a Shanghai industrial park (Hu [6]; Pymnts [7]). 
Existing studies on drone delivery optimization problems usually focus on tactical-level drone facility location problems (Scott and Scott [8]; Hong et al. [2]; Shavarani et al. [9]), and operational-level drone routing problems (Murray and Chu [10]; Chow [11]; Tavana et al. [12]; Dorling et al. [13]). In the drone delivery systems of JD.com and Ele.me, (drone) service routes are designated and fixed and the optimization of drone fleet deployment and planning (DFDP) decisions can greatly improve the delivery efficiency. On each service route, to ensure a safe and ordered flow of the air traffic in the controlled airspace, two consecutive drones departing from the warehouse to deliver parcels must respect a certain time interval (Furini et al. [14]). According to Amazon's drone delivery scheme, the drone operating cost mainly includes the amortized purchasing cost and the maintenance cost, which depend largely on the drone type and number (Keeney [15]). Specifically, during the planning time period, the drone deployment cost is calculated based on leasing (or amortized purchasing) and maintenance cost. Given a fixed number of drones on a route, the drone operating cost is determined by the planned service frequency or the time interval between consecutive departures. Moreover, in order to maintain a goodwill, all parcels must be delivered. Thus, surplus parcel demand (i.e., the number of parcels over drone transportation capacity) will be delivered by couriers with higher labor cost.

Moreover, in the literature, the parcel demand is treated as constant or dynamic (Murray and Chu [10]; Chow [11]; Tavana et al. [12]; Dorling et al. [13]). However, in practice, the number of parcels to be delivered between any two consecutive customers is stochastic due to (i) uncertain market environments, (ii) changing commodity prices, and (iii) intensive competition (Perdikaki et al. [16]). Motivated by the above observations, this paper studies a stochastic drone fleet deployment and planning (DFDP) problem with uncertain customer parcel demand. Service routes are designated and fixed, and each route consists of a sequence of customer stations (called customers for short), which starts from the warehouse and ends at the warehouse, as shown in Figure 1. It is notoriously difficult to find optimal solutions for general stochastic optimization problems (Birge and Louveaux [17]). Therefore, to tackle the difficulty, we employ a two-stage stochastic programming formulation with recourse, which is common in the literature of stochastic problems (Liu et al. [18]).

We borrow the concept of service choice (module) in Francis et al. [19], such that each service module is associated with a combination of departure time intervals (or service frequency) in the planning time period. The problem involves a two-stage decision process: (i) The first stage determines the drone fleet deployment (i.e., the numbers and types of drones) and the drone delivery service module on each route on a tactical level, and (ii) the second stage decides the numbers of parcels delivered by drones and couriers on an operational level. Reducing the system cost has always been an important target to seek from the perspective of company operating. Therefore, the objective in this work is to minimize the total cost, including (i) drone deployment and operating cost and (ii) expected labor cost. The contribution of this paper includes the following:

(1) This paper studies a new stochastic DFDP with uncertain parcel demand, which determines (i) the drone fleet deployment, i.e., the numbers of different types of drones deployed, (ii) the drone service module, and (iii) the numbers of parcels delivered by drones and couriers under each scenario of demand.

(2) For the problem, a novel two-stage stochastic programming formulation is proposed and a classic sample average approximation (SAA) method is first employed. Since SAA is very time-consuming, a hybrid genetic algorithm (GA) is further developed to achieve computational efficiency.

(3) A case study based on the delivery service of Ele.me in Shanghai Jinshan industrial park in China demonstrates the applicability of the proposed methods. Computational results show that, under a given number of scenarios, the hybrid GA outperforms SAA in terms of total computational time with high solution quality. 


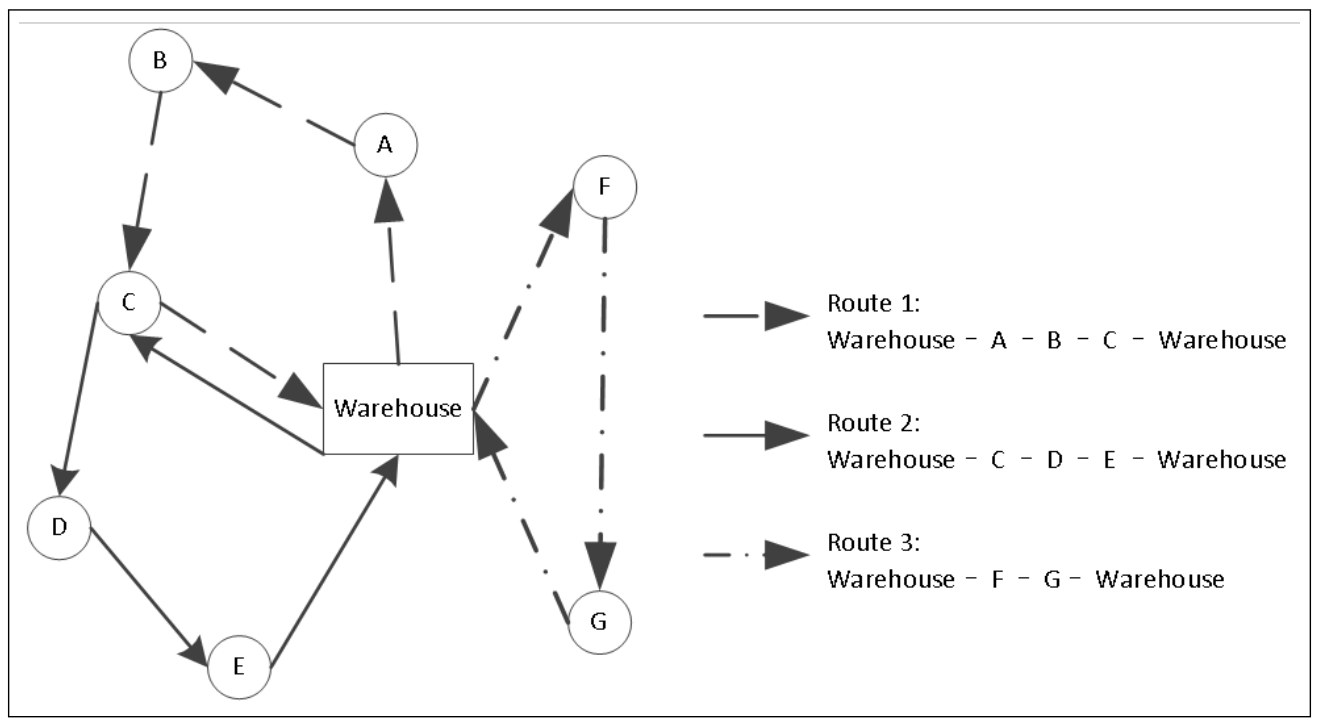

Figure 1. An illustrative example.

The remainder of this paper is organized as follows. Section 2 gives a brief literature review. In Section 3, the problem is described and a two-stage stochastic programming formulation is proposed. In Section 4, a classic SAA method is applied and a hybrid genetic algorithm (GA) is further developed. A case study is conducted, and the computational results are reported and analyzed in Section 5. Section 6 summarizes this paper and suggests future research directions.

\section{Literature Review}

Numerous studies have been conducted to overcome the regulatory and technological difficulties for the widespread adoption of drones (Guenard et al. [20]; McGonigle et al. [21]; Dunford et al. [22]; Baluja et al. [23]; Herissé et al. [24]; Mozaffari et al. [25]). However, since this study falls within the scope of the stochastic DFDP in multiple-type parcel delivery service, only the most related studies are reviewed for brevity. In the following, previous studies on operational-level drone routing problems are first reviewed. We then review the works on tactical-level drone facility location problems. At last, as the vessel fleet deployment problem in liner shipping networks is similar to the studied problem, previous related studies are also briefly reviewed.

\subsection{Operational-Level Drone Routing Problem}

Existing works on the operational-level drone routing problems usually consider the generalization of deterministic vehicle routing problems (VRPs). Murray and Chu [10] introduced a flying sidekick traveling salesman problem (TSP). In the problem, a drone works to collaborate a traditional delivery truck, where the drone can be launched from the truck to distribute parcels. Two mixed-integer linear programming formulations are proposed to minimize the total travel time. Tavana et al. [12] studied a delivery problem where drones can be used for direct transportation and trucks can be used through a standard cross-docking process. The problem is to minimize the total cost of allocation and the time of scheduling simultaneously. An epsilon-constraint method is adapted to solve the problem. Dorling et al. [13] investigated two VRPs for drone delivery by considering the effect of battery and payload weight, i.e., one minimizing total cost with travel time limited and one minimizing the total delivery time with weight restricted. Simulated annealing (SA) algorithms were proposed. Ulmer and Thomas [26] investigated a delivery problem with heterogeneous fleet of drones and vehicles, where goods are delivered either by a drone or by a regular transportation vehicle. They revealed that (i) geographical districting is highly effective in increasing the expected number of deliveries and that (ii) a combination of drone and vehicle fleets may reduce costs significantly. Campbell et al. [27] studied the hybrid truck-drone delivery with drone delivery as an alternative of 
traditional delivery by trucks. They proposed several models to minimize the travel cost and travel time of truck-drone delivery and truck-only delivery. They found that (1) hybrid truck-drone delivery has the potential to provide substantial cost savings, especially in suburban areas, and that (2) the benefits from hybrid truck-drone delivery depend largely on the operating and stopping costs for trucks and drones and on the spatial density of customers. Agatz et al. [28] also investigated a flying sidekick TSP. An integer programming formulation was proposed, and several heuristics based on local search and dynamic programming were developed. Venkatachalam et al. [29] proposed a two-stage stochastic programming for routing drones, where the fuel consumed between any two depots is uncertain. The SAA method was applied, and a heuristic was further proposed for addressing the large-scale problem instances.

In summary, literature considering the stochastic customer demand is very rare.

\subsection{Tactical-Level Drone Facility Location and Drone Fleet Deployment Problem}

Studies on the tactical-level drone delivery usually focus on the drone facility location problems, i.e., locating drone launching and recharging stations and drone fleet deployment (Mozaffari et al. [30]). Scott and Scott [8] studied some innovative applications of drones in healthcare, and they proposed two models to locate the central depot of drone deliveries. Hong et al. [2] considered the limited flight distance of drones that are powered by batteries or fuel, and they investigated the design of station locations and the delivery routes. A mixed-integer linear programming formulation and a heuristic were developed. Shavarani et al. [9] investigated a hierarchical drone facility location problem with limited flight distance. The problem is to optimize the number stations and their locations for launching and recharging drones to minimize the total system cost.

Mozaffari et al. [25] considered the deployment of drones as a flying base station to provide the fly wireless communications to a given geographical area. They considered two scenarios, i.e., a static drone and a mobile drone. Sikeridis et al. [31] considered the public safety networks with drones, and they presented a user equipments cluster formation mechanism to determine the optimal position of the drone.

To our best knowledge, the tactical level of the drone fleet deployment and planning (DFDP) problem has not been studied by previous works.

\subsection{Fleet Deployment and Planning Problem}

The studied DFDP is similar to the liner ship deployment problem (LSDP), also called vessel fleet deployment problem (VFDP), in the liner shipping networks (Wang and Meng [32]; Meng and Wang [33]; Wang and Meng, 2015). Wang and Meng [32] studied a liner ship fleet deployment problem with container transshipment operations, where (1) the shipping routes are fixed, (2) the voyage between two consecutive ports on a route is denoted as a leg, and (3) ships visit each port with a weekly service frequency. In this problem, the service frequency is fixed and the number and speed of ships should be determined. Meng and Wang [33] addressed a liner ship fleet deployment problem with week-dependent container shipment demand, where the transit time between a pair of ports is limited. Wang and Meng [34] considered the bunker management for liner shipping networks, where the ship deployment on each shipping route, the planning sailing speed, and the bunker fuel quantity loaded at each refilling port should be decided. The objective of the problem is to minimize the total cost including ship cost, bunker cost, and inventory cost. The difference between the studied DFDP and the LSDP mainly lies in that (1) the service frequency and the corresponding time interval depend on the service module, which should be optimized in our DFDP, and that (2) there are multiple parcel types differing in volumes and weights in our DFDP.

Concluding, to the best of our knowledge, there is no previous study on the stochastic DFDP in multiple-type parcel delivery service. 


\section{Problem Description and Formulation}

In this section, we first describe the stochastic DFDP in multiple-type parcel delivery service and then propose a two-stage stochastic programming for the problem.

\subsection{Problem Description}

Given a set of service routes $\mathcal{R}$ composed of subsets of customers to load parcels, the set of customers served by route $r \in \mathcal{R}$ is denoted by $\mathcal{I}_{r}=\left[1,2, \ldots, N_{r}\right]$, where $N_{r}$ is the total number of customers composing service route $r \in \mathcal{R}$. The flight from the $i$ th customer to the $(i+1)$ th customer is defined as leg $i$ on route $r \in \mathcal{R}$. Note that each service route is a customer rotation, i.e., leg $N_{r}$ denotes the flight between the $N_{r}$ th customer to the first customer. That is, each service route $r \in \mathcal{R}$ starts from and ends at the warehouse, which is also considered as the first customer. There is a set of parcel categories $\mathcal{H}$, and each parcel of category $h \in \mathcal{H}$ is associated with volume $v_{h}$ and weight $w_{h}$. The demand $\xi_{r i}^{h}$ for parcels of each category $h \in \mathcal{H}$ per time unit on each leg $i \in \mathcal{I}_{r}$ of each route $r \in \mathcal{R}$ is stochastic. All demands are represented by an uncertain vector $\xi=\left[\xi_{11}^{1}, \xi_{12}^{1}, \ldots, \xi_{1, N_{1}}^{1}, \xi_{21}^{1}, \ldots, \xi_{2, N_{2}}^{1}, \ldots, \xi_{|\mathcal{R}|, 1}^{1}, \ldots \xi_{|\mathcal{R}|, N_{|\mathcal{R}|}}^{1} \xi_{11}^{2}, \ldots, \xi_{|\mathcal{R}|, N_{|\mathcal{R}|}}^{2}, \ldots, \xi_{|\mathcal{R}|, N_{|\mathcal{R}|}}^{|\mathcal{H}|}\right]^{\top}$, and $\xi \in \Xi$ and $\Xi \subset \mathbb{R}^{\sum_{h \in \mathcal{H}} \sum_{r \in \mathcal{R}} N_{r}}$.

To better understand the problem, an example is illustrated in Figure 1, where seven customers are served by three routes. Customer sequences on the service routes can be represented as follows:

$\begin{array}{ll}r=1, N_{1}=4: & \text { Warehouse } \rightarrow A \rightarrow B \rightarrow C \rightarrow \text { Warehouse } \\ r=2, N_{2}=4: & \text { Warehouse } \rightarrow C \rightarrow D \rightarrow E \rightarrow \text { Warehouse } \\ r=3, N_{3}=3: & \text { Warehouse } \rightarrow F \rightarrow G \rightarrow \text { Warehouse }\end{array}$

There is a set of drone types $\mathcal{L}$ with different volume and weight capacities. After the numbers of different types of drones deployed have been determined, the drone service module on each route should be then decided. Note that each service module corresponds to a certain time interval between two consecutive drones. Figure 2, for example, illustrates two different service modules, where the third route stated above is considered. Figure 2a shows that drones on the route delivery parcels under a service module that corresponds to a larger time interval between two consecutive drones, and Figure $2 b$ presents a drone service module with a smaller time interval between two consecutive drones.

An express company usually first decides (1) the drone fleet deployment, i.e., the number of different types of drones on each route, and (2) the drone service module on each route. Then, under the realized scenario of parcel demand, the second-stage decision, i.e., the number of parcels of each category delivered by drones and couriers, will be determined. The objective is to minimize the sum of (i) drone deployment and operating costs and (ii) the expectation of the labor cost for couriers to deliver parcels in a planning time period.

For the problem, during the planning time period, it is assumed that (i) service routes are designated and fixed, as in line with the practical drone delivery systems of JD.com and Ele.me; (ii) drones deployed on a route are of the same type, as the same ship type on a route in Wang and Meng (2015); (iii) two consecutive drones on each route depart from the warehouse in a certain time interval to ensure a safe and ordered flow of drones; (iv) the time interval or service frequency during the planning time period depend on the service module; (v) the number of parcels of each category is uncertain; and (vi) parcels can be delivered by drones and couriers to ensure all parcels are delivered. 


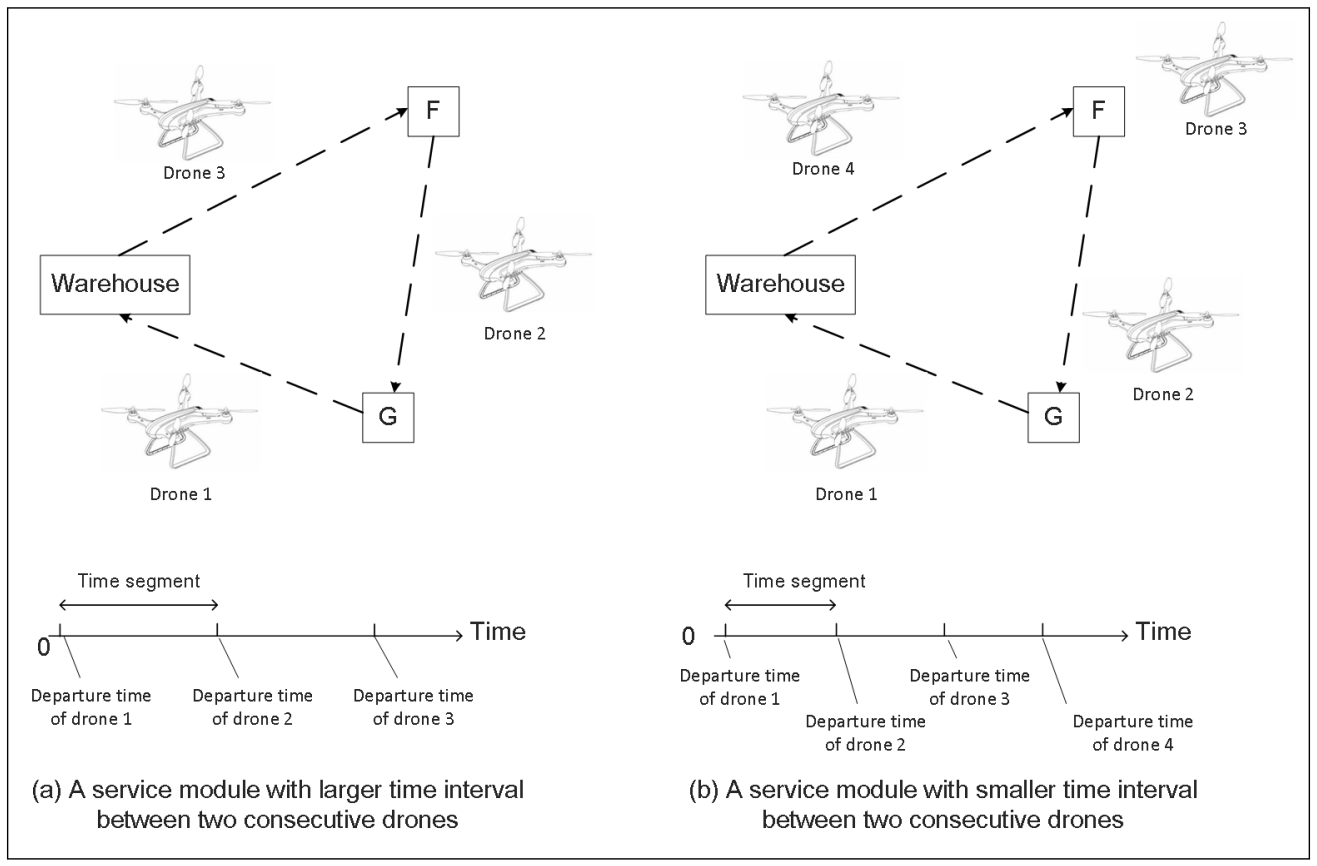

Figure 2. An illustrative example for service module.

\subsection{Formulation}

In the following, we give parameters (shown in Table 1), define decision variables, and present the two-stage stochastic programming formulation for the proposed problem.

Table 1. Parameters.

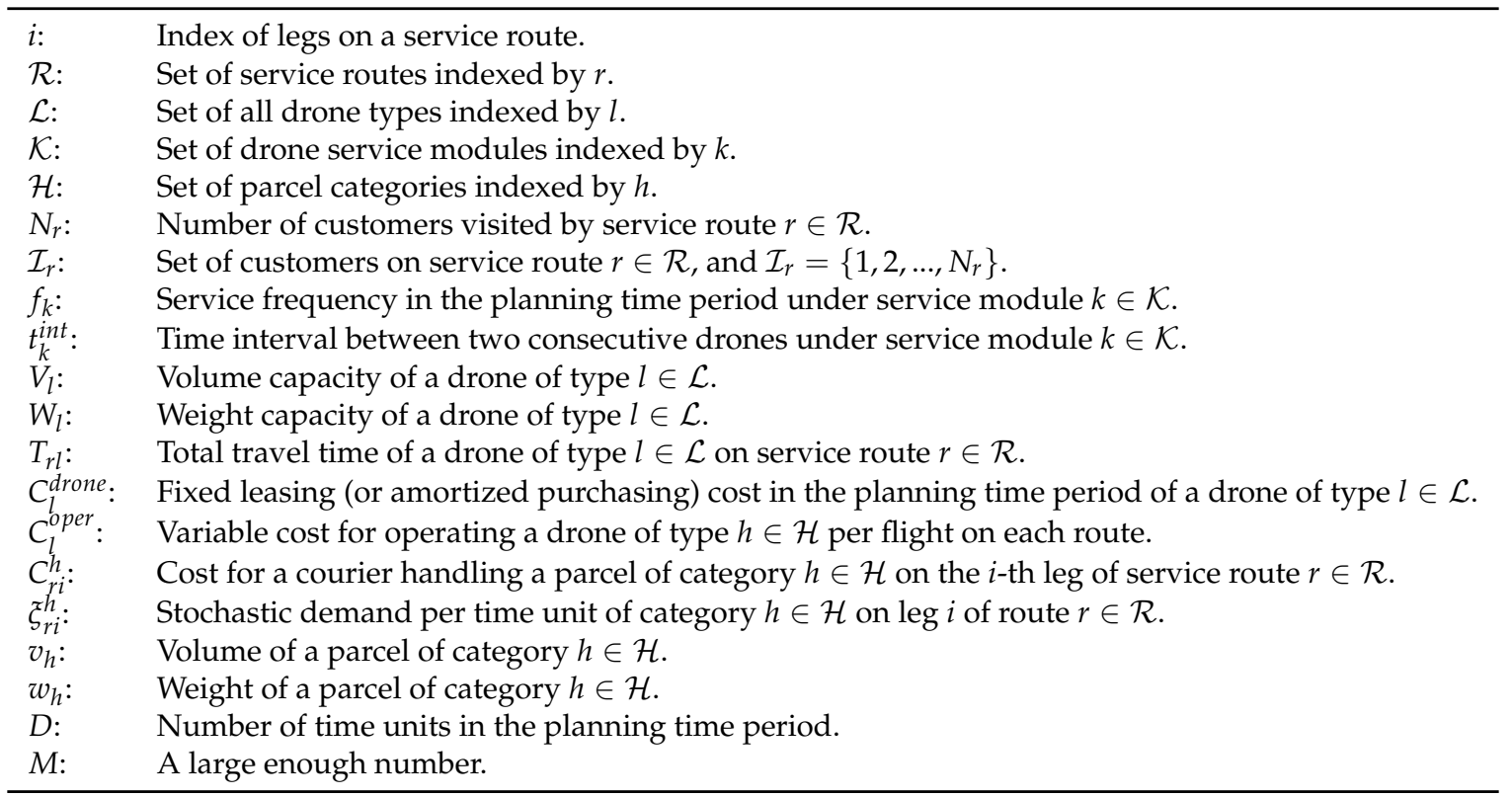

\section{Variables:}

- $x_{r l}$ : Number of drones of type $l \in \mathcal{L}$ deployed on service route $r \in \mathcal{R}$, and $x=$

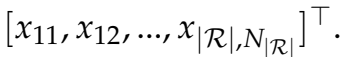

- $\gamma_{r l}$ : A binary variable equal to 1 if there is a drone of type $l \in \mathcal{L}$ deployed on service route $r \in \mathcal{R}$, 0 otherwise, and $\gamma=\left[\gamma_{11}, \gamma_{12}, \ldots, \gamma_{|\mathcal{R}|, N_{|\mathcal{R}|}}\right]^{\top}$. 
- $\quad \mu_{r k}$ : A binary variable equal to 1 if the service module $k \in \mathcal{K}$ is selected on route $r \in \mathcal{R}, 0$ otherwise, and $\boldsymbol{\mu}=\left[\mu_{11}, \mu_{12}, \ldots, \mu_{|\mathcal{R}|,|\mathcal{K}|}\right]^{\top}$.

- $\quad y_{r i}^{h}(\mathcal{\xi})$ : Number of parcels of category $h \in \mathcal{H}$ transported by drones on leg $i \in \mathcal{I}_{r}$ of route $r \in \mathcal{R}$ under realized $\xi \in \Xi$.

- $\quad v_{r l k}$ : A nonnegative integral variable used to linearize $x_{r l} \cdot \mu_{r k}$, and $v_{r l k}=x_{r l} \cdot \mu_{r k}$.

- $\quad z_{r k i}^{h}(\xi)$ : A nonnegative integral variable used to linearize $\mu_{r k} \cdot y_{r i}^{h}(\xi)$, and $z_{r k i}^{h}(\xi)=\mu_{r k} \cdot y_{r i}^{h}(\xi)$, $\forall \tilde{\xi} \in \Xi$.

- $Q(x, \gamma, \boldsymbol{\mu}, \boldsymbol{\xi})$ : The recourse function value, i.e., the labor cost, with given $x, \gamma$ and $\mu$ under realized $\xi \in \Xi$ during the planning time period.

As the done deployment and service module selection are considered as the tactical decisions, decision variables $x_{r l}, \gamma_{r l}, \mu_{r k}$, and $v_{r l k}$ do not depend on the realization of $\xi$. Two-stage stochastic programming formulation (P1) for the problem can be expressed as follows:

(P1):

$$
\begin{array}{ll}
\min & \sum_{r \in R} \sum_{l \in \mathcal{L}}\left(C_{l}^{\text {drone }} x_{r l}+\sum_{k \in \mathcal{K}} C_{l}^{\text {oper }} x_{r l} \mu_{r k}\right)+\mathbb{E}_{\tilde{\zeta} \in \Xi}[Q(x, \gamma, \mu, \xi)] \\
\text { s.t. } \quad & x_{r l} \geq \gamma_{r l}, \quad \forall r \in \mathcal{R}, l \in \mathcal{L} \\
& x_{r l} \leq M \cdot \gamma_{r l}, \quad \forall r \in \mathcal{R}, l \in \mathcal{L} \\
& \sum_{l \in \mathcal{L}} \gamma_{r l}=1, \quad \forall r \in \mathcal{R} \\
& \sum_{k \in \mathcal{K}} \mu_{r k}=1, \quad \forall r \in \mathcal{R} \\
& \sum_{k \in \mathcal{K}} t_{k}^{i n t} \cdot x_{r l} \cdot \mu_{r k} \geq \sum_{l \in \mathcal{L}} T_{r l} \cdot \gamma_{r l}, \quad \forall r \in \mathcal{R} \\
& v_{r l k} \leq x_{r l}, \quad \forall r \in \mathcal{R}, l \in \mathcal{L}, k \in \mathcal{K} \\
& v_{r l k} \leq M \cdot \mu_{r k}, \quad \forall r \in \mathcal{R}, l \in \mathcal{L}, k \in \mathcal{K} \\
& v_{r l k} \geq x_{r l}-M \cdot\left(1-\mu_{r k}\right), \quad \forall r \in \mathcal{R}, l \in \mathcal{L}, k \in \mathcal{K} \\
& \sum_{k \in \mathcal{K}} t_{k}^{i n t} \cdot v_{r l k} \geq \sum_{l \in \mathcal{L}} T_{r l} \cdot \gamma_{r l}, \quad \forall r \in \mathcal{R}, l \in \mathcal{L} \\
& \gamma_{r l}, \mu_{r k} \in\{0,1\}, \quad \forall r \in \mathcal{R}, l \in \mathcal{L}, k \in \mathcal{K} \\
& x_{r l}, v_{r l k}, \in \mathbb{Z}^{+}, \quad \forall r \in \mathcal{R}, l \in \mathcal{L}, k \in \mathcal{K}
\end{array}
$$

where

$$
\begin{aligned}
Q(x, \gamma, \boldsymbol{\mu}, \boldsymbol{\xi})= & \min \left\{\sum_{r \in \mathcal{R}} \sum_{i \in \mathcal{I}_{r}} \sum_{h \in \mathcal{H}}\left(D \cdot \xi_{r i}^{h}(\boldsymbol{\xi})-\sum_{k \in \mathcal{K}} f_{k} \cdot z_{r k i}^{h}(\boldsymbol{\xi})\right) \cdot C_{r i}^{h} \mid\right. \\
& y_{r i}^{h}(\boldsymbol{\xi}) \leq \sum_{k \in \mathcal{K}} t_{k}^{i n t} \cdot \mu_{r k} \cdot \xi_{r i}^{h}, \quad \forall r \in \mathcal{R}, i \in \mathcal{I}_{r}, h \in \mathcal{H}, \xi \in \Xi \\
& \sum_{h \in \mathcal{H}} v_{h} \cdot y_{r i}^{h}(\boldsymbol{\xi}) \leq \sum_{l \in \mathcal{L}} V_{l} \cdot \gamma_{r l}, \quad \forall r \in \mathcal{R}, i \in \mathcal{I}_{r}, \xi \in \Xi \\
& \sum_{h \in \mathcal{H}} w_{h} \cdot y_{r i}^{h}(\boldsymbol{\xi}) \leq \sum_{l \in \mathcal{L}} W_{l} \cdot \gamma_{r l}, \quad \forall r \in \mathcal{R}, i \in \mathcal{I}_{r}, \xi \in \Xi \\
& z_{r k i}^{h}(\boldsymbol{\xi}) \leq y_{r i}^{h}(\boldsymbol{\xi}), \quad \forall r \in \mathcal{R}, i \in \mathcal{I}_{r}, h \in \mathcal{H}, k \in \mathcal{K}, \xi \in \Xi \\
& z_{r k i}^{h}(\boldsymbol{\xi}) \leq M \cdot \mu_{r k}, \quad \forall r \in \mathcal{R}, i \in \mathcal{I}_{r}, h \in \mathcal{H}, k \in \mathcal{K}, \xi \in \Xi \\
& z_{r k i}^{h}(\boldsymbol{\xi}) \geq y_{r i}^{h}(\boldsymbol{\xi})-M \cdot\left(1-\mu_{r k}\right), \quad \forall r \in \mathcal{R}, i \in \mathcal{I}_{r}, h \in \mathcal{H}, k \in \mathcal{K}, \xi \in \Xi \\
& \left.y_{r i}^{h}(\boldsymbol{\xi}), z_{r k i}^{h}(\boldsymbol{\xi}) \in \mathbb{Z}^{+}, \quad \forall r \in \mathcal{R}, i \in \mathcal{I}_{r}, l \in \mathcal{L}, k \in \mathcal{K}, \xi \in \Xi\right\}
\end{aligned}
$$


The objective function of Equation (1) is to minimize the sum of drone deployment and operating costs and the expectation of labor cost during the planning time period. The constraints of of Equations (2)-(4) ensure that drones deployed on each route are of the same type. The constraint of Equation (5) ensures that only one service module can be selected for a service route. The constraint of Equation (6) guarantees the time interval between any two consecutive drones during the planning time period. The constraints of Equations (7)-(10) focus on linearizing the constraint of Equation (6). The constraints of Equations (11) and (12) give the domains of the first-stage decision variables.

$Q(x, \gamma, \mu, \xi)$ denotes the second-stage labor cost under each realized vector $\xi$ of demands, satisfying the constraints of Equations (13)-(19). The constraint of Equation (13) implies that the number of parcels of each category delivered by drones should not exceed the demand cumulated during the time interval. The constraints of Equations (14) and (15) respect the volume capacity and the weight capacity of each drone. The constraints of Equations (16)-(18) aim to linearize $z_{r k i}^{h}(\boldsymbol{\xi})=y_{r i}^{h}(\boldsymbol{\xi})$. The constraints of Equation (19) gives the restrictions on the second-stage decision variables.

\section{Solution Approaches}

It is a challenging task to obtain the optimal solution (Birge and Louveaux, 2011). Besides, as the set $\Xi$ of all possible vectors of demands can be infinite, it is difficult to solve formulation $\mathbf{P 1}$ by calling the off-the-shelf solvers. Therefore, in this section, based on the idea of SAA, we first propose an approximated SAA-based formulation, which can be solved by calling commercial solvers. As exactly solving the SAA-based model is rather time-consuming, a hybrid GA is further developed, to obtain feasible solutions in a reasonable time for the SAA-based formulation.

\section{1. $S A A$}

Sample average approximation (SAA) is a relatively popular and widely applied solution approach for solving large stochastic programming problems. SAA is based on the Monte Carlo simulation and deterministic optimization techniques (Kleywegt et al. [35]; Pagnoncelli et al. [36]; Ralph and $\mathrm{Xu}$ [37]). Based on the historical data, the empirical mean values and standard deviation of demands can be calculated. The basic idea is to employ a finite set of scenarios $\Omega$, which are assumed to be independent identically distributed (iid) sampling of the uncertain vector $\xi$. Under the set $\Omega$ of scenarios, the SAA approximates the expected demand-related cost by a sample average estimation such that the transformed problem can be well-addressed by deterministic techniques (Verweij et al. [38]). Accordingly, an approximated deterministic SAA-based formulation $\mathbf{P 2}$ is proposed, in which the second-stage decisions depend on the first-stage decisions and on the realized demands under each scenario $\omega \in \Omega$. SAA method is easy to implement and performs well under a sufficient number of scenarios (Wang and Ahmed [39]).

In the following, we present a formulation (P2) with the purpose to apply the SAA method, where $\omega \in \Omega$ denotes the index of scenarios. 
(P2):

$$
\begin{array}{ll}
\min & \sum_{r \in R} \sum_{l \in \mathcal{L}}\left(C_{l}^{d r o n e} x_{r l}+\sum_{k \in \mathcal{K}} C_{l}^{o p e r} x_{r l} \mu_{r k}\right)+\frac{1}{|\Omega|} \sum_{\omega \in \Omega} \sum_{r \in \mathcal{R}} \sum_{i \in \mathcal{I}_{r}} \sum_{h \in \mathcal{H}}\left(D \xi_{r i}^{h}(\omega)-\sum_{k \in \mathcal{K}} f_{k} z_{r k i}^{h}(\omega)\right) C_{r i}^{h} \\
\text { s.t. } & (2)-(11) \\
& y_{r i}^{h}(\omega) \leq \sum_{k \in \mathcal{K}} t_{k}^{i n t} \cdot \mu_{r k} \cdot \xi_{r i}^{h}(\omega), \quad \forall r \in \mathcal{R}, i \in \mathcal{I}_{r}, h \in \mathcal{H}, \omega \in \Omega \\
& \sum_{h \in \mathcal{H}} v_{h} \cdot y_{r i}^{h}(\omega) \leq \sum_{l \in \mathcal{L}} V_{l} \cdot \gamma_{r l}, \quad \forall r \in \mathcal{R}, i \in \mathcal{I}_{r}, \omega \in \Omega \\
& \sum_{h \in \mathcal{H}} w_{h} \cdot y_{r i}^{h}(\omega) \leq \sum_{l \in \mathcal{L}} W_{l} \cdot \gamma_{r l}, \quad \forall r \in \mathcal{R}, i \in \mathcal{I}_{r}, \omega \in \Omega \\
& z_{r k i}^{h}(\omega) \leq y_{r i}^{h}(\omega), \quad \forall r \in \mathcal{R}, i \in \mathcal{I}_{r}, h \in \mathcal{H}, k \in \mathcal{K}, \omega \in \Omega \\
& z_{r k i}^{h}(\omega) \leq M \cdot \mu_{r k}, \quad \forall r \in \mathcal{R}, i \in \mathcal{I}_{r}, h \in \mathcal{H}, k \in \mathcal{K}, \omega \in \Omega \\
& z_{r k i}^{h}(\omega) \geq y_{r i}^{h}(\omega)-M \cdot\left(1-\mu_{r k}\right), \quad \forall r \in \mathcal{R}, i \in \mathcal{I}_{r}, h \in \mathcal{H}, k \in \mathcal{K}, \omega \in \Omega \\
& y_{r i}^{h}(\omega), z_{r k i}^{h}(\omega) \in \mathbb{Z}^{+}, \quad \forall r \in \mathcal{R}, i \in \mathcal{I}_{r}, l \in \mathcal{L}, \omega \in \Omega
\end{array}
$$

Formulation P2 can be exactly solved by calling commercial optimization softwares, such as CPLEX. The obtained solution includes the first-stage decisions and the second-stage decisions under given scenarios set $\Omega$. The obtained first-stage solution, i.e., $x, \gamma$ and $\mu$, can be considered as an approximately optimal first-stage drone fleet deployment, including the type, number, and service choice of drones deployed on each route, of the original problem. When the number of given scenarios $|\Omega|$ is sufficiently large and $|\Omega| \rightarrow \infty$, the optimal objective value of $\mathbf{P} 2$ converges to the true optimal objective value almost surely (Bertsimas et al. [40]).

\subsection{Hybrid GA}

It can be observed from the computational results that the SAA method is quite time-consuming, even under a small set of scenarios $\Omega$. Therefore, it is necessary to develop solution methods to achieve computational efficiency. Metaheuristics such as GA and hybrid GA seem to be quite suited, as they focus on processing a set of parallel solutions and exploiting similarities between solutions in order to obtain the (near) optimal solutions in the solution space (Kalayci et al. [41]). In this subsection, we propose a hybrid GA based on a heuristic rule.

GA is first introduced in Holland [42] and is based on the biological reproduction rules. GA starts with a set of initial feasible solutions. In each iteration, offspring solutions are generated via crossover and mutation procedures, and then, the population including the current solutions and offspring solutions will be renewed according to their objective values. The algorithm stops when a stopping criterion is reached. In the following, we present the hybrid GA for the studied stochastic DFDP in multiple-type parcel delivery service.

\subsubsection{Coding}

In the developed hybrid GA for the studied stochastic optimization problem, each solution is represented by a chromosome (individual) composed of three parts, as shown in Figure 3, and the three gene parts denote drone type selection, the drone number, and service module: (i) The first part is the selection of drone type on each route, where the number at the $r$ th position denotes the type of drones, (ii) the drone number on each route, and (iii) the drone service module on each route. When the type, number, and service module of drones deployed on each route are determined, under each scenario, parcels of each customer on each route are handled by drones and couriers via a heuristic rule. The heuristic rule is based on the idea that parcels with higher labor cost and smaller volume and weight are delivered by drones. The heuristic rule consists of the following steps: (1) Sort the 
parcels in decreasing order of $\frac{C_{j}}{v_{j} \cdot w_{j}}$, where $j$ denotes the index of parcels, $C_{j}$ denotes the labor cost for parcel $j$ delivered by courier, and $v_{j}$ and $w_{j}$ denote the volume and weight of parcel $j$; (2) assign parcels to drones in the obtained sequence with respect to the volume and weight capacities of each drone; and (3) parcels that are not assigned to drones will be delivered by couriers.

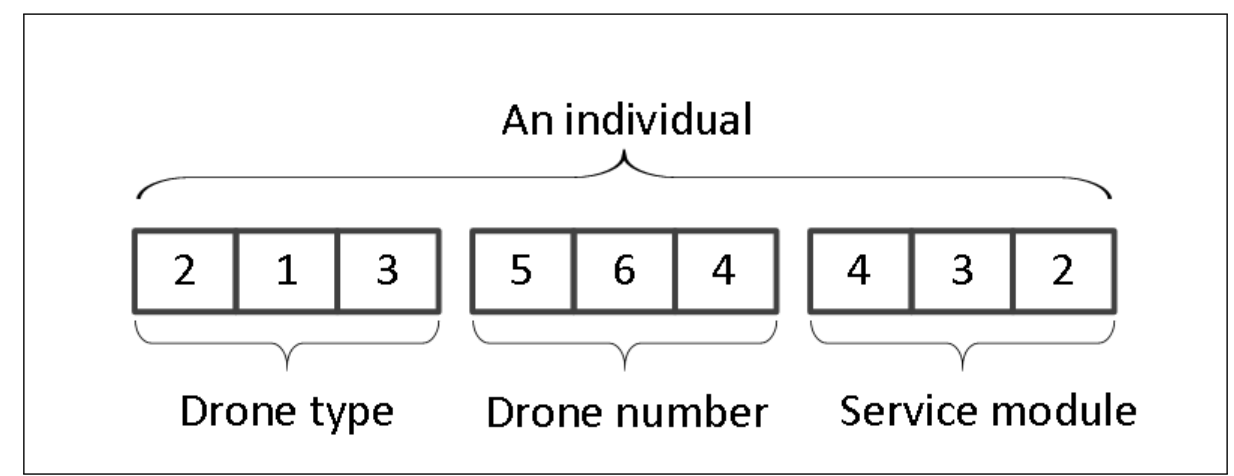

Figure 3. Coding (parcels are assigned to drones and couriers via the heuristic rule).

For the generation of an initial individual, firstly the type of drones on each route is randomly selected. The drone number and drone service module on each route are randomly generated. If the constraint of Equation (6) is violated for a service route, then the drone number and service module will be regenerated. Since parcels are assigned to drones and couriers via the heuristic rule under each scenario, the sum of drone operating cost and the expectation of the labor cost of couriers can be calculated accordingly.

\subsubsection{Crossover and Mutation}

During each iteration, offspring solutions are reproduced by current individuals via genetic operators, i.e., the crossover and mutation operations. For the crossover operation, the identical drone types of two parent individuals will be copied to the two offspring solutions. For each service route, if the drone types selected in two parent solutions are the same, then the drone type will be still selected for the route in the offsprings and the corresponding number and service module of drones will be not changed in the offsprings. Otherwise, the type, number, and service module of drones deployed on each route will be reselected randomly for the offsprings. Figure 4 illustrates the crossover procedure. The identical drone types of two parent solutions are labelled with a circle in dotted line, and the type, number, and service of drones on this route are copied to the offspring individuals. Moreover, the type, number, and service module of drones on route 2 and route 3 are randomly generated.

There are two ways for mutation: (1) random modification of the number of drones and (2) random reselection of the service module of drones deployed on each route. The first mutation method is shown in Figure 5; the first mutation method consists of (i) copying the type, number, and service module of drones deployed on each route to the offspring individual; (ii) randomly selecting a service route; and (iii) randomly modifying the number of drones on this route. The second mutation method is illustrated in Figure 6 and includes (i) copying the genetic parts to the offspring solution, (ii) randomly selecting a service route, and (iii) randomly modifying the service module of drones deployed on this route. Then, under each scenario, parcels are assigned to drones and couriers via the heuristic rule. 


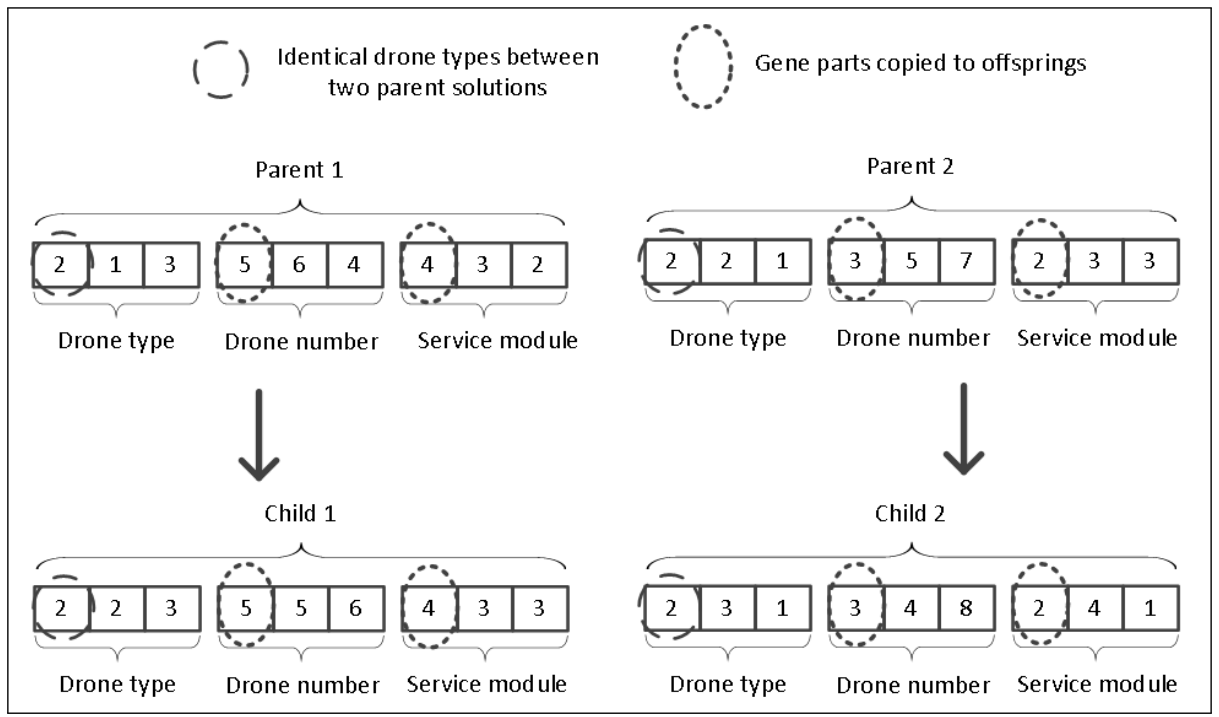

Figure 4. Crossover (reproducing the identical genetic parts between parents to offsprings).

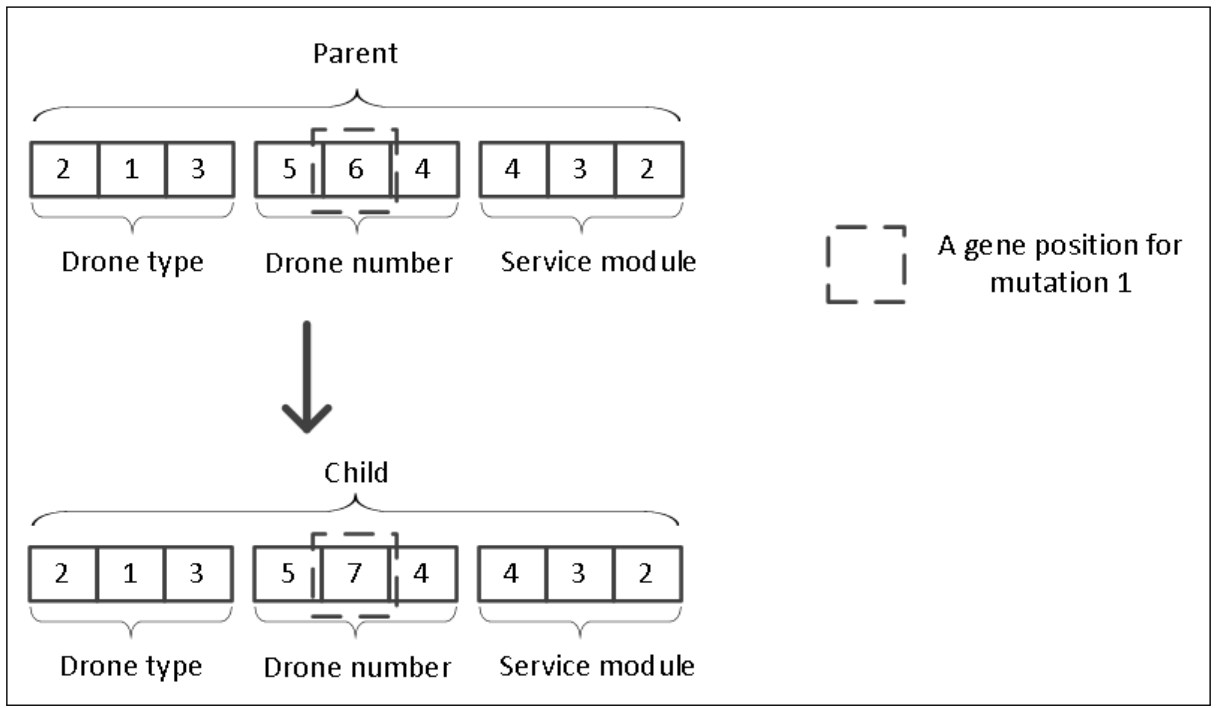

Figure 5. Mutation 1 (random modification of drone numbers).

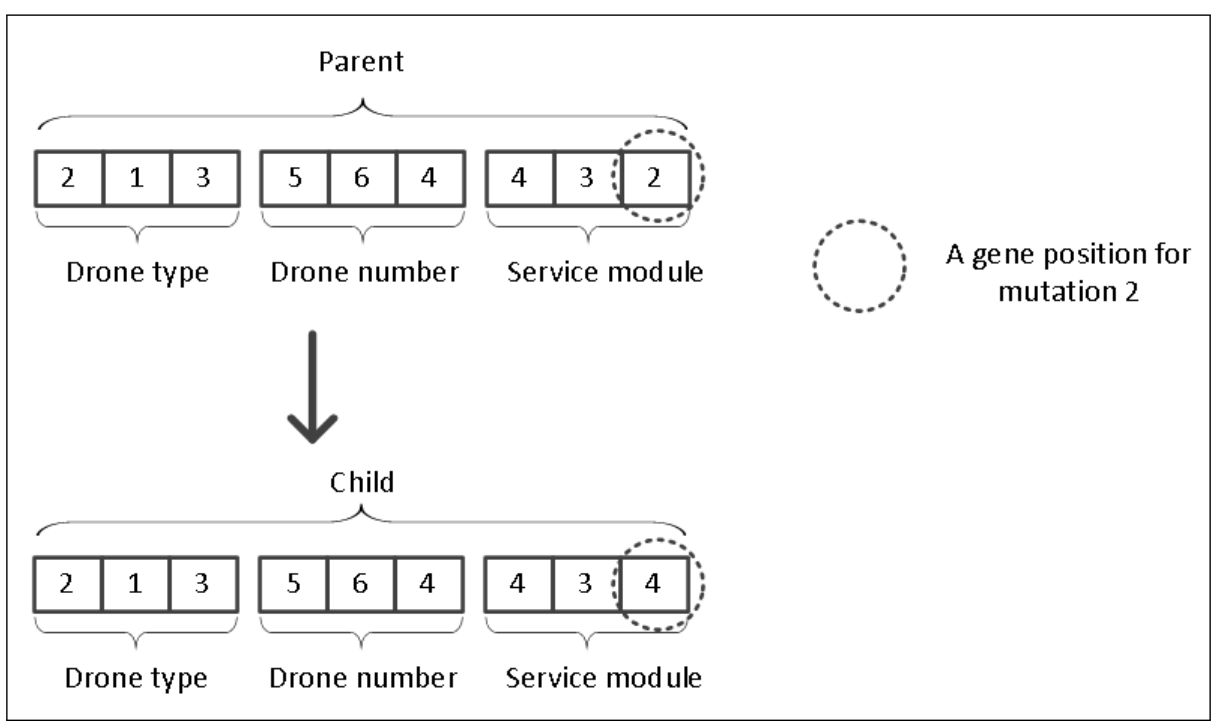

Figure 6. Mutation 2 (random modification of drone service modules). 


\section{Computational Experiments}

In this section, we evaluate the performance of proposed solution approaches by conducting a case study based on the Shanghai Jinshan industrial park network. Both the SAA method and the hybrid GA are coded in MATLAB_2014b, andthe SAA method is combined with CPLEX 12.6. All computational experiments are conducted on a personal computer with a $3.60 \mathrm{GHz}$ processor and 8.00 GB RAM under the Windows 7 operating system. The total computational times of both proposed solution approaches are limited to $3600 \mathrm{~s}$.

For the hybrid GA, a preliminary analysis has been conducted in order to fine-tune the parameters, which are reported in Table 2. Population size and maximum iteration number are set to be 50 and 20 respectively.

Table 2. Parameters for hybrid genetic algorithm (GA).

\begin{tabular}{cc}
\hline Parameter & Value (Hybrid GA) \\
\hline Population size & 50 \\
Generation number & 20 \\
Crossover probability & 0.9 \\
Mutation 1 probability & 0.2 \\
Mutation 2 probability & 0.8 \\
\hline
\end{tabular}

\subsection{Data Generation}

We conduct a case study based on the drone delivery information of Ele.me to evaluate the applicability of the proposed solution methods. Ele.me has developed a fleet of drones to delivery food on fixed routes in Shanghai Jinshan industrial park. Based on the transportation and the geographical location information of Shanghai Jinshan industrial park, a network is proposed. The network has a total of 20 customers, as shown in Figure 7. There are 11 service routes, three types of drones, and 4 categories of parcels, as shown in Tables 3-5, respectively. Note that the total travel time on each route depends on the drone speed and the total route distance. The planning time period is set to be one week, i.e., $168 \mathrm{~h}(10,080 \mathrm{~min})$. There are 4 service modules for drones with departure time intervals, i.e., 5, 10, 15 and $20 \mathrm{~min}$ with corresponding service frequencies calculated as $f_{k}=\frac{10080}{t_{k}^{i n t}}, \forall k \in \mathcal{K}$. The labor cost for a courier delivering a parcel on each leg of each route depends on the distance of the leg. Moreover, we assume the total number of scenarios to be 50. Under each scenario, the number of parcels of each category on each leg of each route per minute is uniformly generated from 1 to 3 .

Table 3. Service routes.

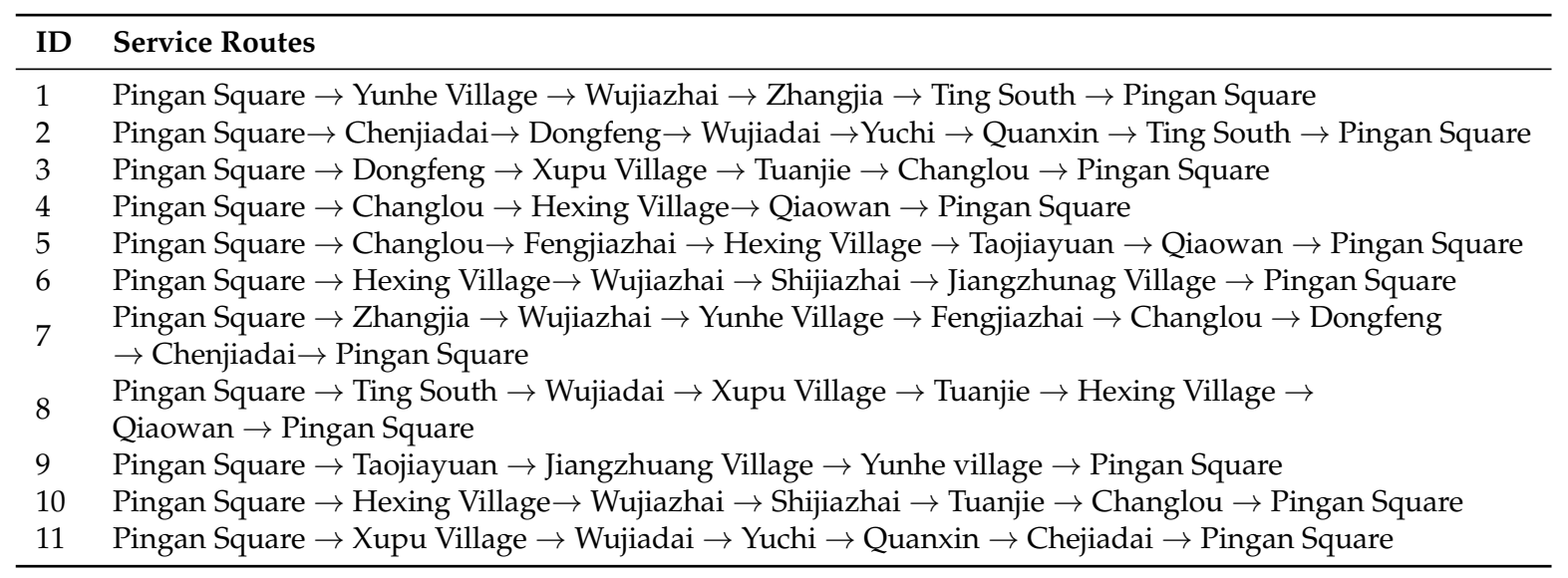


Table 4. Drone types.

\begin{tabular}{ccccc}
\hline Drone Type & \multicolumn{2}{c}{ Capacity } & & \\
Volume $\left.\mathbf{( m}^{\mathbf{3}}\right)$ & Weight $\mathbf{( k g )}$ & $\begin{array}{c}\text { Operating Cost } \\
\text { (Yuan) }\end{array}$ & $\begin{array}{c}\text { Average Speed } \\
\mathbf{( k m} / \mathbf{h})\end{array}$ \\
\hline 1 & 1 & 30 & 350 & 20 \\
2 & 2 & 35 & 420 & 30 \\
3 & 3 & 40 & 490 & 40 \\
\hline
\end{tabular}

Table 5. Parcel categories.

\begin{tabular}{cccc}
\hline Parcel Category & Volume $\left.\mathbf{( m}^{\mathbf{3}}\right)$ & Weight $\mathbf{( k g )}$ & $\begin{array}{l}\text { Labor Cost Per Parcel } \\
\text { Per kilometer (yuan) }\end{array}$ \\
\hline 1 & 0.01 & 1 & 0.2 \\
2 & 0.02 & 2 & 0.4 \\
3 & 0.01 & 2 & 0.3 \\
4 & 0.02 & 1 & 0.3 \\
\hline
\end{tabular}

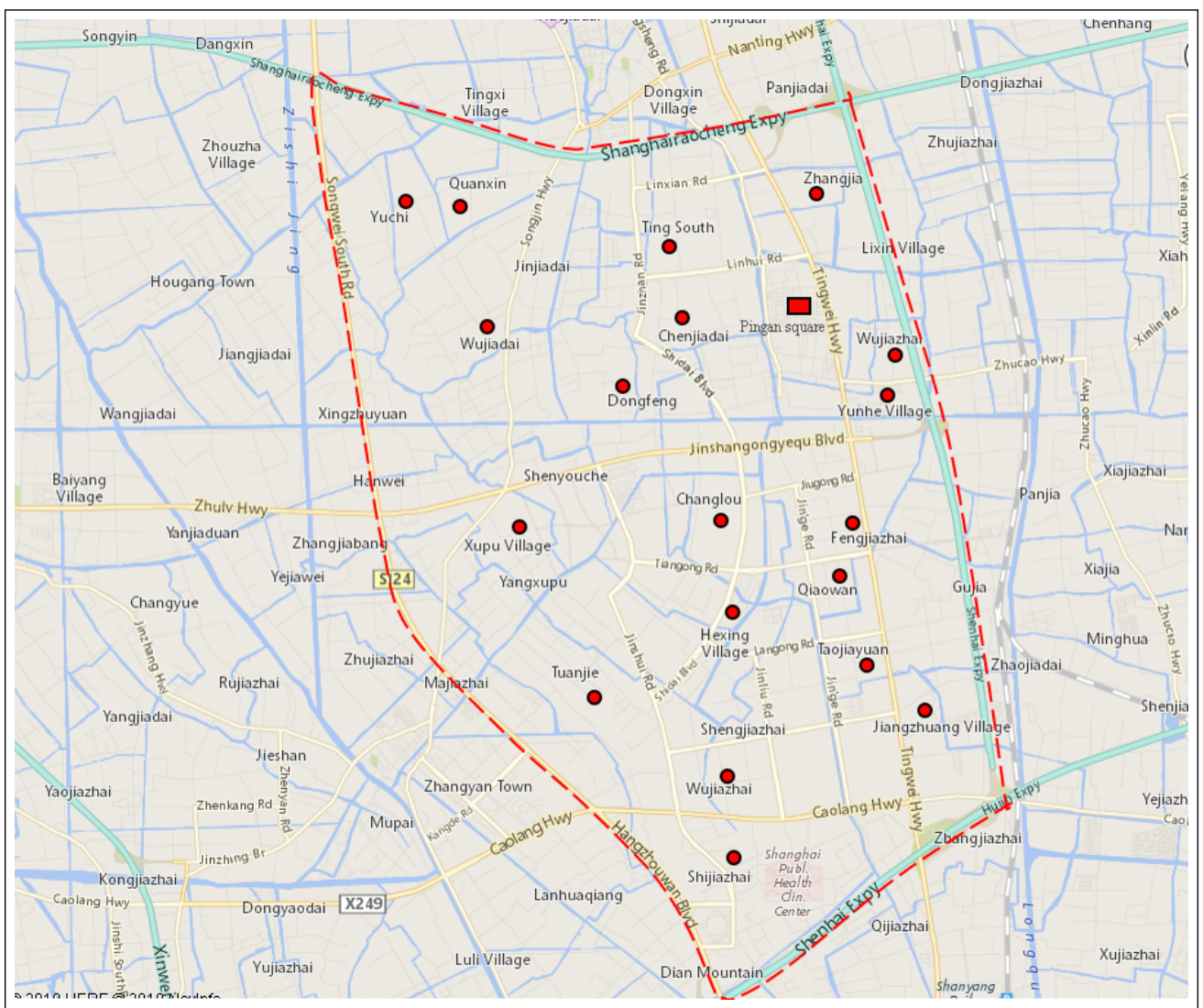

Figure 7. Shanghai Jinshan industrial park drone delivery network.

\subsection{Computational Results}

The computational experiments are conducted and reported in this part. For the two solution methods, after the drone fleet deployment is determined, the objective value is calculated as the sum of the drone leasing and operating costs and the expected labor cost (under all 50 scenarios). Note that the scenario set $\Omega$ comprises 50 scenarios and that we evaluate the objective value based on the entire $\Omega$.

An illustrative example based on a small network is first studied to compare the proposed two solution method with the deterministic situation. Note that the deterministic situation is considered where the demand $\xi_{r i}^{h}$ is fixed and equal to its mean value. In the example, there are 6 customers 
and 3 drone routes, $\mathcal{I}_{1}=[1,2,6,5,1,2,1], \mathcal{I}_{2}=[1,2,3,2,1]$, and $\mathcal{I}_{3}=[2,4,1,2]$. The computational results are reported in Table 6 , where $N_{\text {drone }}$ denotes the number of drones deployed in total. Note that drones selected by the three methods are of type 3 . We can obtain that the number obtained under the deterministic situation is 5003, which is 103 and 16 larger than those obtained by the SAA and the HGA. That is, considering the uncertain demand is realistic and cost-saving.

Table 6. An illustrative example.

\begin{tabular}{ccccccc}
\hline \multirow{2}{*}{ Route } & \multicolumn{2}{c}{ Deterministic Situation } & \multicolumn{2}{c}{ SAA } & \multicolumn{2}{c}{ HGA } \\
& $N_{\text {drone }}$ & Service module & $N_{\text {drone }}$ & Service Module & $N_{\text {drone }}$ & Service Module \\
\hline 1 & 2 & 2 & 4 & 1 & 4 & 1 \\
2 & 2 & 2 & 3 & 1 & 3 & 1 \\
3 & 2 & 2 & 3 & 1 & 3 & 1 \\
\hline Obj & 5003 & & 4900 & & 4987 \\
\hline
\end{tabular}

The performance of the proposed solution methods under different number of scenarios is tested, and the results are reported in Table 7. Scenarios for the solution methods are randomly selected from the given 50 scenarios. The number of scenarios for the solution methods range from 3 to 50 . From Table 7, we observe that the SAA obtains a similar objective with the deterministic situation. Moreover, it can be obtained that the computational time of SAA increases dramatically with the number of scenarios and that SAA loses its power to solve the problem within $3600 \mathrm{~s}$ if there are 25 scenarios. That is because, when the number of scenarios increases, the number of decision variables and constraints increase rapidly. The average computational time of SAA is $1424.2 \mathrm{~s}$, which is about 9 times larger than that of the hybrid GA. Besides, as the scale of the tested instance is not large, the drone deployment decisions obtained by SAA and the hybrid GA are very similar. The average objective value of the hybrid GA is $6.9913 \times 10^{5}$, which is about $0.98 \%$ larger than that of SAA. In summary, (i) for each test, the quality of solutions obtained by SAA and the hybrid GA is very similar, (ii) SAA is relatively time-consuming and the computational time of SAA increases rapidly with the number of scenarios, and (iii) the average computational time of the hybrid GA is quite smaller than that of SAA.

Table 7. Impact of the number of scenarios $|\Omega|$.

\begin{tabular}{|c|c|c|c|c|c|c|}
\hline$|\Omega|$ & $N_{\text {drone }}$ & $\begin{array}{c}\text { SAA } \\
\text { Obj }\left(\times 10^{5}\right)\end{array}$ & Time (s) & $N_{\text {drone }}$ & $\begin{array}{c}\text { HGA } \\
\text { Obj }\left(\times 10^{5}\right)\end{array}$ & Time (s) \\
\hline 3 & 50 & 7.3531 & 56.0 & 50 & 7.5165 & 48.2 \\
\hline 5 & 50 & 6.3544 & 119.3 & 50 & 6.3795 & 66.9 \\
\hline 10 & 50 & 6.8960 & 462.7 & 50 & 7.0713 & 79.8 \\
\hline 15 & 50 & 6.9026 & 2883.2 & 50 & 6.9867 & 89.5 \\
\hline 20 & 50 & 7.1125 & 3600.0 & 50 & 7.1602 & 112.4 \\
\hline 25 & - & - & - & 50 & 7.0799 & 138.6 \\
\hline 30 & - & - & - & 50 & 7.0001 & 164.2 \\
\hline 35 & - & - & - & 50 & 6.9762 & 189.4 \\
\hline 40 & - & - & - & 50 & 6.8648 & 249.8 \\
\hline 50 & - & - & - & 50 & 6.8782 & 308.7 \\
\hline Average & 50 & 6.9237 & 1424.2 & 50 & 6.9913 & 144.8 \\
\hline Lower bound & 50 & 6.3544 & 56.0 & 50 & 6.3795 & 48.2 \\
\hline Upper bound & 50 & 7.3531 & 3600.0 & 50 & 7.5165 & 308.7 \\
\hline
\end{tabular}

The impact of the total flight time of drones on each route is examined and reported in Table 8 . Since the flight time of a drone on each route depends on the distance and the average speed, the increase of average drone speed is set from $0,5,10, \ldots, 30 \mathrm{~km} / \mathrm{h}$. Based on the analysis on the tradeoff between the solution quality and the computational time, the number of scenarios for SAA is set to be 10 and that for the hybrid GA is set to be 50 . For SAA, 10 distinct scenarios are randomly generated from $\Omega$. After the first-stage decisions, i.e., the drone fleet deployment and drone service 
module, are determined, the expected labor cost is calculated as the sample average of the labor costs under 50 scenarios. From Table 8, it can be obtained that the number of drones deployed and the objective values decrease when the average speed increases. That may be because, when the average drone speed increases, the total flight time of a drone on each route decreases and the number of drones deployed on each route may decrease in ensuring the constraint of Equation (9). Therefore, the drone operating cost decreases accordingly. Besides, the average computational times of SAA and the hybrid GA are 467.7 and 312.7 seconds, respectively.

Table 8. The impact of the total flight time of drones on each route.

\begin{tabular}{|c|c|c|c|c|c|c|}
\hline \multirow{2}{*}{$\begin{array}{c}\text { Increase of the } \\
\text { Average Drone Speed }\end{array}$} & \multicolumn{3}{|c|}{ SAA with 10 Scenarios } & \multicolumn{3}{|c|}{ HGA with 50 Scenarios } \\
\hline & $N_{\text {drone }}$ & Time (s) & $\operatorname{Obj}\left(\times 10^{5}\right)$ & $N_{\text {drone }}$ & Time (s) & $\operatorname{Obj}\left(\times 10^{5}\right)$ \\
\hline 0 & 50 & 463.2 & 6.8960 & 50 & 312.7 & 6.8782 \\
\hline 5 & 44 & 465.3 & 6.2485 & 44 & 306.3 & 6.8488 \\
\hline 10 & 42 & 468.9 & 6.1849 & 42 & 317.9 & 6.8390 \\
\hline 15 & 39 & 472.1 & 6.2458 & 39 & 317.2 & 6.8243 \\
\hline 20 & 35 & 471.6 & 6.2346 & 36 & 318.3 & 6.8047 \\
\hline 25 & 34 & 465.8 & 6.1793 & 34 & 306.6 & 6.7998 \\
\hline 30 & 30 & 466.9 & 6.1345 & 31 & 309.9 & 6.7802 \\
\hline Average & 39.1 & 467.7 & 6.3034 & 39.4 & 312.7 & 6.8250 \\
\hline
\end{tabular}

The impact of the volume and weight capacities of drones are tested and presented in Table 9. We increase the volume and weight capacities by four combinations, i.e., $(0.5,5),(0.5,10),(1,5)$, and $(1,10)$. It can be observed that the number of drones deployed decreases when the volume and weight capacities increase. That may be because, when the capacities increases, the number of parcels can be delivered by a drone increases and thus the time interval may increase. Therefore, the number of drones deployed may decrease in ensuring the constraint of Equation (9) and the objective value may decrease accordingly, as shown in Table 9.

Table 9. The impact of the volume and weight capacities of drones.

\begin{tabular}{ccccccc}
\hline Increase of Volume & \multicolumn{3}{c}{ SAA with 10 Scenarios } & \multicolumn{3}{c}{ HGA with 50 Scenarios } \\
and Weight Capacities & $\boldsymbol{N}_{\text {drone }}$ & Time $(\mathbf{s})$ & $\mathbf{O b j}\left(\times \mathbf{1 0}^{\mathbf{5}}\right)$ & $\boldsymbol{N}_{\text {drone }}$ & Time $(\mathbf{s})$ & $\mathbf{O b j}\left(\times \mathbf{1 0}^{\mathbf{5}}\right)$ \\
\hline$(0,0)$ & 50 & 467.5 & 6.8960 & 50 & 313.3 & 6.8782 \\
$(0.5,5)$ & 37 & 472.6 & 6.7510 & 37 & 311.0 & 6.8145 \\
$(0.5,10)$ & 37 & 468.9 & 6.2560 & 37 & 309.8 & 6.8145 \\
$(1,5)$ & 37 & 469.8 & 6.2346 & 36 & 320.4 & 6.8047 \\
$(1,10)$ & 37 & 470.6 & 6.1793 & 37 & 322.7 & 6.7998 \\
\hline Average & 39.6 & 469.9 & 6.4634 & 39.4 & 315.4 & 6.8223 \\
\hline
\end{tabular}

The sensitivity of the value of time intervals $t_{k}^{\text {int }}$ of each service module is then tested. We reduce the time interval of each service module by $1,2,3$, and 4, respectively. It can be observed from Table 10 that, when the time interval values decrease, the number of drones deployed increases. That may be because, when the time intervals decrease, the number of drones deployed increases to guarantee the constraint of Equation (9), the number of parcels delivered by drones decreases, and thus the number of parcels handled by couriers decreases. With the decrease of the time intervals, the objective value first decreases and then increases. That may be because there is a tradeoff between the number of drones deployed and the labor cost; when the number of drones on each route is too large, the drone operating cost increases rapidly. 
Table 10. The impact of time interval values.

\begin{tabular}{ccccccc}
\hline $\begin{array}{c}\text { Decrease of } \\
\text { Time Intervals }\end{array}$ & \multicolumn{3}{c}{ SAA with 10 Scenarios } & \multicolumn{3}{c}{ HGA with 50 Scenarios } \\
& $N_{\text {drone }}$ & Time (s) & Obj $\left(\times \mathbf{1 0}^{\mathbf{5}}\right)$ & N $_{\text {drone }}$ & Time (s) & Obj $\left(\times \mathbf{1 0}^{\mathbf{5}}\right)$ \\
\hline 0 & 50 & 466.1 & 6.8960 & 50 & 314.6 & 6.8782 \\
1 & 62 & 464.2 & 2.4349 & 62 & 310.4 & 2.9725 \\
2 & 81 & 478.1 & 0.9162 & 81 & 313.4 & 1.2973 \\
3 & 117 & 472.9 & 0.5733 & 117 & 319.5 & 0.5769 \\
4 & 229 & 469.8 & 1.1221 & 228 & 320.3 & 1.1221 \\
\hline Average & 107.8 & 470.22 & 2.3885 & 107.6 & 315.6 & 2.5694 \\
\hline
\end{tabular}

In summary, from the computational results reported in Tables 6-10, it can be obtained that

(1) with the increase of the number of scenarios, the computational time of SAA increases dramatically;

(2) given the same number of scenarios, the computational time of the hybrid GA is smaller than SAA with high solution quality;

(3) with the increase of average drone speed and the total flight time, the number of drones deployed and the total cost decrease;

(4) when the volume and weight capacities of drones increase, the number of drones deployed and the total cost decrease;

(5) when the time intervals decrease, the number of drones deployed increases; and

(6) the developed hybrid GA outperforms the SAA in terms of the computational time with high solution quality.

Therefore, for the stochastic drone fleet deployment and planning problem in multiple-type parcel delivery service, we recommend applying SAA when the number of scenarios is small and applying the hybrid GA method when the number of scenarios is large.

\section{Conclusions}

This work studies the stochastic drone fleet deployment and planning problem in multiple-type parcel delivery service. In the problem, the drone fleet deployment, i.e., the type, number, and service module of drones deployed on each route, should be decided with uncertain parcel demand. Under each realized scenario, the number of parcels delivered by drones and couriers should be determined. The objective is to minimize the sum of the drone leasing and operating costs and the expected labor cost. A novel two-stage stochastic programming formulation is proposed. To solve the problem, a classic SAA is applied and a hybrid GA is further developed to achieve computational efficiency.

Future research directions may include (i) designing heuristics that can solve the problem more efficiently; (ii) considering more practical conditions, such as the situation where the labor cost function is more complicated; (iii) considering heterogeneous drones on each drone route; and (iv) studying the problem with labor cost as a piecewise or convex function.

Author Contributions: conceptualization, M.L.; methodology, X.L.; data curation, M.Z.; writing-original draft preparation, X.L.; writing - review and editing, F.Z.

Funding: This work was supported by the National Natural Science Foundation of China (NSFC) under Grants 71531011, 71771048, and 71571134. This work was also supported by the Fundamental Research Funds for the Central Universities.

Acknowledgments: The authors would like to thank the anonymous referees for their constructive comments. The authors would also like to thank Feng Chu for her efforts to improve the quality of our work.

Conflicts of Interest: The authors declare no conflict of interest. The funders had no role in the design of the study; in the collection, analyses, or interpretation of data; in the writing of the manuscript; or in the decision to publish the results. 


\section{References}

1. Sikeridis, D.; Tsiropoulou, E.E.; Devetsikiotis, M.; Papavassiliou, S. Wireless powered Public Safety IoT: A UAV-assisted adaptive-learning approach towards energy efficiency. J. Netw. Comput. Appl. 2018, 123, 69-79. [CrossRef]

2. Hong, I.; Kuby, M.; Murray, A.T. A range-restricted recharging station coverage model for drone delivery service planning. Transp. Res. Part Emerg. Technol. 2018, 90, 198-212. [CrossRef]

3. Zhang, C.; Kovacs, J.M. The application of small unmanned aerial systems for precision agriculture: A review. Precis. Agric. 2012, 13, 693-712. [CrossRef]

4. $\quad$ Aleem, Z. The Chinese Version of Amazon Is Already Using Drones to Deliver Stuff to Customers; Vox Media, Inc.: New York, NY, USA, 2017.

5. Rose, C. Amazon's Jeff Bezos looks to the future. CBS News, 1 December 2013.

6. $\mathrm{Hu}, \mathrm{Y}$. Your takeaway order in Shanghai might soon be delivered by a drone. Urban Family, 31 May 2018.

7. Pymnts. Ele.me Clared to Use Food Delivery Drones in China. 2018. Available online: https:/ /www.pymnts. com/news/delivery/2018/eleme-food-delivery-drones-china/ (accessed on 31 May 2018)

8. Scott, J.; Scott, C. Drone delivery models for healthcare. In Proceedings of the 50th Hawaii International Conference on System Sciences, Waikoloa Village, HI, USA, 4-7 January 2017. [CrossRef]

9. Shavarani, S.M.; Nejad, M.G.; Rismanchian, F.; Izbirak, G. Application of hierarchical facility location problem for optimization of a drone delivery system: a case study of Amazon prime air in the city of San Francisco. Int. J. Adv. Manuf. Technol. 2018, 95, 3141-3153. [CrossRef]

10. Murray, C.C.; Chu, A.G. The flying sidekick traveling salesman problem: Optimization of drone-assisted parcel delivery. Transp. Res. Part Emerg. Technol. 2015, 54, 86-109. [CrossRef]

11. Chow, J.Y. Dynamic UAV-based traffic monitoring under uncertainty as a stochastic arc-inventory routing policy. Int. J. Transp. Sci. Technol. 2016, 5, 167-185. [CrossRef]

12. Tavana, M.; Khalili-Damghani, K.; Santos-Arteaga, F.J.; Zandi, M.H. Drone shipping versus truck delivery in a cross-docking system with multiple fleets and products. Expert Syst. Appl. 2017, 72, 93-107. [CrossRef]

13. Dorling, K.; Heinrichs, J.; Messier, G.G.; Magierowski, S. Vehicle routing problems for drone delivery. IEEE Trans. Syst. Man Cybern. Syst. 2016, 47, 70-85. [CrossRef]

14. Furini, F.; Persiani, C.A.; Toth, P. The time dependent traveling salesman planning problem in controlled airspace. Transp. Res. Part Methodol. 2016, 90, 38-55. [CrossRef]

15. Keeney. How Can Amazon Charge $\$ 1$ for Drone Delivery. 2015. Available online: https://ark-invest.com/ research/drone-delivery-amazon (accessed on 5 May 2015).

16. Perdikaki, O.; Kostamis, D.; Swaminathan, J.M. Timing of service investments for retailers under competition and demand uncertainty. Eur. J. Oper. Res. 2016, 254, 188-201. [CrossRef]

17. Birge, J.R.; Louveaux, F. Introduction to Stochastic Programming; Springer: New York, NY, USA, 2011.

18. Liu, M.; Liu, X.; Zhang, E.; Chu, F.; Chu, C. Scenario-based heuristic to two-stage stochastic program for the parallel machine ScheLoc problem. Int. J. Prod. Res. 2019, 57, 1706-1723. [CrossRef]

19. Francis, P.; Smilowitz, K.; Tzur, M. The period vehicle routing problem with service choice. Transp. Sci. 2006, 40, 439-454. [CrossRef]

20. Guenard, N.; Hamel, T.; Mahony, R. A practical visual servo control for an unmanned aerial vehicle. IEEE Trans. Robot. 2008, 24, 331-340. [CrossRef]

21. McGonigle, A.; Aiuppa, A.; Giudice, G.; Tamburello, G.; Hodson, A.; Gurrieri, S. Unmanned aerial vehicle measurements of volcanic carbon dioxide fluxes. Geophys. Res. Lett. 2008, 35. [CrossRef]

22. Dunford, R.; Michel, K.; Gagnage, M.; Piégay, H.; Trémelo, M.L. Potential and constraints of Unmanned Aerial Vehicle technology for the characterization of Mediterranean riparian forest. Int. J. Remote Sens. 2009, 30, 4915-4935. [CrossRef]

23. Baluja, J.; Diago, M.P.; Balda, P.; Zorer, R.; Meggio, F.; Morales, F.; Tardaguila, J. Assessment of vineyard water status variability by thermal and multispectral imagery using an unmanned aerial vehicle (UAV). Irrig. Sci. 2012, 30, 511-522. [CrossRef]

24. Herissé, B.; Hamel, T.; Mahony, R.; Russotto, F.X. Landing a VTOL unmanned aerial vehicle on a moving platform using optical flow. IEEE Trans. Robot. 2011, 28, 77-89. [CrossRef]

25. Mozaffari, M.; Saad, W.; Bennis, M.; Debbah, M. Unmanned aerial vehicle with underlaid device-to-device communications: Performance and tradeoffs. IEEE Trans. Wirel. Commun. 2016, 15, 3949-3963. [CrossRef] 
26. Ulmer, M.W.; Thomas, B.W. Same-day delivery with heterogeneous fleets of drones and vehicles. Networks 2018, 72, 475-505. [CrossRef]

27. Campbell, J.F.; Sweeney, D.; Zhang, J. Strategic Design for Delivery with Trucks and Drones; Supply Chain Analytics Report SCMA; SCMA-2017-0201; University of Missouri-St. Louis: St. Louis, MO, USA, 2017.

28. Agatz, N.; Bouman, P.; Schmidt, M. Optimization approaches for the traveling salesman problem with drone. Transp. Sci. 2018, 52, 965-981. [CrossRef]

29. Venkatachalam, S.; Sundar, K.; Rathinam, S. Two-stage stochastic programming model for routing multiple drones with fuel constraints. arXiv 2017, arXiv:1711.04936.

30. Mozaffari, M.; Saad, W.; Bennis, M.; Debbah, M. Mobile unmanned aerial vehicles (UAVs) for energy-efficient internet of things communications. IEEE Trans. Wirel. Commun. 2017, 16, 7574-7589. [CrossRef]

31. Sikeridis, D.; EleniTsiropoulou, E.; Devetsikiotis, M.; Papavassiliou, S. Self-Adaptive Energy Efficient Operation in UAV-Assisted Public Safety Networks. In Proceedings of the 2018 IEEE 19th International Workshop on Signal Processing Advances in Wireless Communications (SPAWC), Kalamata, Greece, 25-28 June 2018; pp. 1-5.

32. Wang, S.; Meng, Q. Liner ship route schedule design with sea contingency time and port time uncertainty. Transp. Res. Part B 2012, 46, 615-633. [CrossRef]

33. Meng, Q.; Wang, S. Liner ship fleet deployment with week-dependent container shipment demand. Eur. J. Oper. Res. 2012, 222, 241-252. [CrossRef]

34. Wang, S.; Meng, Q. Robust bunker management for liner shipping networks. Eur. J. Oper. Res. 2015, 243, 789-797. [CrossRef]

35. Kleywegt, A.J.; Nori, V.S.; Savelsbergh, M.W.P. The Stochastic Inventory Routing Problem with Direct Deliveries. Transp. Sci. 2002, 36, 94-118. [CrossRef]

36. Pagnoncelli, B.K.; Ahmed, S.; Shapiro, A. Sample Average Approximation Method for Chance Constrained Programming: Theory and Applications. J. Optim. Theory Appl. 2009, 142, 399-416. [CrossRef]

37. Ralph, D.; $\mathrm{Xu}, \mathrm{H}$. Convergence of stationary points of sample average two-stage stochastic programs: A generalized equation approach. Math. Oper. Res. 2011, 36, 568-592. [CrossRef]

38. Verweij, B.; Ahmed, S.; Kleywegt, A.J.; Nemhauser, G.; Shapiro, A. The sample average approximation method applied to stochastic routing problems: a computational study. Comput. Optim. Appl. 2003, 24, 289-333. [CrossRef]

39. Wang, W.; Ahmed, S. Sample average approximation of expected value constrained stochastic programs. Oper. Res. Lett. 2008, 36, 515-519. [CrossRef]

40. Bertsimas, D.; Gupta, V.; Kallus, N. Robust sample average approximation. Math. Program. 2017, 171, $217-282$. [CrossRef]

41. Kalayci, C.B.; Polat, O.; Gupta, S.M. A hybrid genetic algorithm for sequence-dependent disassembly line balancing problem. Ann. Oper. Res. 2016, 242, 321-354. [CrossRef]

42. Holland, J.H. Adaptation in natural and artificial systems. Q. Rev. Biol. 1975, 6, 126-137.

(C) 2019 by the authors. Licensee MDPI, Basel, Switzerland. This article is an open access article distributed under the terms and conditions of the Creative Commons Attribution (CC BY) license (http://creativecommons.org/licenses/by/4.0/). 